\title{
Germinación y efectos del almacenamiento de frutos de Prosopis ruscifolia (Fabaceae)
}

\author{
Germination and effects of storage of fruits of Prosopis ruscifolia (Fabaceae)
}

\author{
Abdala Nelly Roxana ${ }^{a *}$, Sandra Bravo a, Marcia Acosta ${ }^{\text {a }}$ \\ *Autor de correspondencia: ${ }^{a}$ Universidad Nacional de Santiago del Estero, Facultad de Ciencias Forestales, \\ Instituto de Silvicultura y Manejo de Bosques, Avenida Belgrano (s) 1912, Santiago del Estero, Argentina, \\ tel.: +540385 4509550, yeny@unse.edu.ar
}

\begin{abstract}
SUMMARY
Prosopis ruscifolia is a promising forest species for restoration of saline areas that produces high quality wood and nutraceutical interest fruits. Its germination requirements and optimal storage conditions are unknown. The objectives of the work were 1) to evaluate different methods to stimulate germination, 2) to establish if there is an interaction between pregerminative treatments and the substrate used in laboratory tests (paper or sand), and 3) to analyze the effect of three different conditions of fruit storage on germination. Normal seedlings (PG), abnormal seedlings, hard and dead seeds were recorded. Three fruit samples were stored for eight months at $7 \pm 2{ }^{\circ} \mathrm{C},-17 \pm 2{ }^{\circ} \mathrm{C}$ and the control at $25^{\circ} \mathrm{C}$. Afterwards, germination tests were carried out and the speed indexes (IVG) and mean germination time (TMG) were determined. Results indicated that chemical scarification with sulfuric acid was the most efficient pregerminative treatment to stimulate germination in both substrates. Immersion in water at room temperature showed the lowest percentage of germination in both substrates. The seeds of fruits stored at $7 \pm 2{ }^{\circ} \mathrm{C}$ had the highest germination percentage. Low temperatures improved velocity indices (IVG) and mean germination time (TMG). Results indicated the need for methods to overcome seed dormancy in P. ruscifolia and the excellent tolerance to low temperatures during fruit storage.
\end{abstract}

Key words: dormancy, seed test, storage, vinal.

\section{RESUMEN}

Prosopis ruscifolia es una especie forestal promisoria para restauración de áreas salinas, que produce madera de calidad y frutos de interés nutraceútico. Sus requerimientos germinativos y condiciones de almacenamiento óptimas no se han establecido aún. Los objetivos del trabajo fueron 1) evaluar diferentes métodos para estimular la germinación, 2) establecer si hay interacción entre los tratamientos pregerminativos y el sustrato empleado en ensayos de laboratorio (papel o arena), y 3) analizar el efecto de tres diferentes condiciones de almacenamiento de los frutos sobre la germinación. Se registraron plántulas normales (PG), plántulas anormales, semillas duras y muertas. Tres muestras de frutos se almacenaron durante ocho meses a $7 \pm 2{ }^{\circ} \mathrm{C},-17 \pm 2{ }^{\circ} \mathrm{C}$ y el control a $25^{\circ} \mathrm{C}$. Luego se realizaron ensayos de germinación y determinaron los índices de velocidad (IVG) y tiempo medio de germinación (TMG). Los resultados indicaron que la escarificación química con ácido sulfúrico fue el tratamiento pregerminativo más eficiente para estimular la germinación en ambos sustratos. La inmersión en agua a temperatura ambiente mostró el menor porcentaje de germinación en ambos sustratos. Las semillas de los frutos almacenados a $7 \pm 2{ }^{\circ} \mathrm{C}$ presentaron el máximo porcentaje de germinación. Las bajas temperaturas mejoraron los índices de velocidad (IVG) y tiempo medio de germinación (TMG). Los resultados indicaron la necesidad de métodos para superar la dormición de las semillas en P. ruscifolia y la excelente tolerancia a bajas temperaturas durante el almacenamiento de los frutos.

Palabras clave: dormición, análisis de semillas, almacenamiento, vinal.

\section{INTRODUCCIÓN}

Las especies de Prosopis son comunes en ambientes áridos y semiáridos de América, donde representan recursos genéticos valiosos por los servicios ambientales que brindan (Villagra y Álvarez 2019). Es sabido que dichas regiones experimentan fuertes procesos de degradación, con pérdidas crecientes de recursos forestales, que afectan la producción de bienes y servicios ecosistémicos (Villagra et al. 2010). La recuperación productiva de áreas degrada- das incluye planes de forestación y la implementación de sistemas de uso múltiple como los silvopastoriles. Prosopis ruscifolia Griseb. conocida como "vinal", es una especie leñosa nativa del Chaco semiárido y subhúmedo que en los últimos años se ve revalorizada tanto por la calidad de su madera, como por el valor forrajero y alimenticio de sus frutos y semillas (González Galán et al. 2008). Su tolerancia a la salinidad (Meloni et al. 2008, Taleisnik y López Launestein 2011), la convierte en una especie promisoria desde el punto de vista productivo ya que permite el desarrollo 
de la actividad ganadera, con buena producción de pasturas bajo su cubierta (Astrada y Adámoli 2005). Se la propone además como pie de injerto de otras especies de Prosopis que son sensibles a la salinidad (Felker et al. 2000).

La germinación y el establecimiento de las plántulas es un proceso crítico en estos ambientes áridos y semiáridos, donde hay un marcado déficit hídrico y las plantas están expuestas a condiciones climáticas adversas a lo largo de varios meses. Las especies de Prosopis se propagan habitualmente de manera sexual, por lo cual es importante conocer cuáles son los métodos más adecuados para promover la germinación. En general, se considera que las fabáceas poseen semillas con dormición física debido a la presencia de sustancias hidrófobas en la cutícula, de macroescleridas en empalizada o ambas características a nivel de la cubierta seminal (Abraham de Noir et al. 2004, Ferreira y Borghetti 2004, Bravo et al. 2011, Villarreal Garza et al. 2013, Baskin y Baskin 2014). Lopes de Oliveira et al. (2017) sostienen que la dormición impuesta por la cubierta seminal es parte de una estrategia de supervivencia y perpetuación de las especies.

Entre los tratamientos pregerminativos más utilizados para superar la dormición física, se encuentran la escarificación mecánica, térmica y la química (Sanabria et al. 2004, Dutra et al. 2007, Muñoz et al. 2009, Pereira et al. 2016, Trombin Souza et al. 2018). Algunos autores describen los requerimientos germinativos de diferentes especies de Prosopis (Bravo et al. 2011, Sobrevilla Solís et al. 2013, Rodríguez Araujo et al. 2017) y sugieren la importancia del estudio particular para cada especie y ambiente. Los métodos para estimular la germinación de $P$. ruscifolia es información necesaria para planes de manejo en plantación. Así también, la evaluación del comportamiento germinativo en diferentes sustratos es un tópico escasamente abordado en trabajos de esta temática con especies forestales. Son escasos los trabajos donde se mencionan diferencias en el número de plántulas normales germinadas en distintos sustratos (Bravo et al. 2011, Lopes de Oliveira et al. 2017). Estos datos son de gran importancia al momento de evaluar en laboratorio la calidad de lotes de semillas, sobre todo en especies no domesticadas. Antecedentes en otras especies de Prosopis indican que presentan dormición impuesta por la cubierta seminal (Bravo et al. 2011, Sobrevilla-Solís et al. 2013, Rodríguez Araujo et al. 2017). La distribución de la humedad en el sustrato de siembra y la presencia de compuestos tóxicos pueden alterar la germinación e inclusive el número de plántulas normales en un lote de semillas (Bravo et al. 2011, ISTA 2015, Lopes de Oliveira et al. 2017).

Las semillas de Prosopis spp. se comportan como ortodoxas en el almacenamiento (Bonner 2008). La maduración de los frutos de esta especie se concentra durante diciembre y enero dentro de su área de distribución (Abraham de Noir y Bravo 2014). La producción de plantines en almácigos o contenedores habitualmente se realiza a partir de octubre, por lo cual los frutos suelen almacenarse durante varios meses previos a la siembra. La liberación de las semillas del fruto y la selección de aquellas que reúnen las características deseables de calidad, es una labor que demanda mucho tiempo y costos. El almacenamiento de frutos y semillas de esta especie, así como de otras especies de Prosopis, presenta dificultades debido al ataque de lepidópteros, coleópteros y hemípteros (Mazzuferi y Conles 2005). Una alternativa que disminuye las pérdidas por estos ataques es el almacenamiento de los frutos a largo plazo a temperaturas entre 0 y $20^{\circ} \mathrm{C}$ (Piotto y Fallieri 2001, Rojas Espinoza 2004, Were et al. 2004). La germinación, el porcentaje de plántulas normales y los índices de germinación se reducen con un incremento de la humedad y de la temperatura de almacenamiento (Azadi y Younesi 2013). Se necesitan investigaciones tendientes a conocer las condiciones óptimas de almacenamiento de frutos de esta especie y su tolerancia a temperaturas bajo cero.

Aun cuando presenta mayores dificultades logísticas (espacio, tamizado, esterilización, entre otros), la arena como sustrato es recomendado para algunas especies que presentan inconvenientes en la germinación en papel (Dousseau et al. 2011, ISTA 2015). El presente trabajo plantea las siguientes hipótesis: a) las semillas de P. ruscifolia presentan dormición impuesta por la cubierta seminal, que puede superarse con la aplicación de procedimientos pregerminativos que disminuyen su dureza y/o interrumpen la barrera de macroesclereidas, b) el sustrato en el que se realizan las pruebas de germinación en laboratorio influye en el número de semillas germinadas con plántulas normales, y c) el almacenamiento de frutos de esta especie a temperaturas inferiores a $7{ }^{\circ} \mathrm{C} \mathrm{y}-17^{\circ} \mathrm{C}$ no afecta el porcentaje de germinación.

Los objetivos de este trabajo son: 1) evaluar diferentes tratamientos pregerminativos para superar la dormición de las semillas en P. ruscifolia; 2) establecer si hay interacción entre los tratamientos pregerminativos (inmersión en ácido sulfúrico, corte a nivel de cubierta seminal seminal, escarificación física, inmersión en agua a temperatura ambiente y en agua caliente) y el sustrato empleado en ensayos de laboratorio (papel o arena), y 3 ) analizar el efecto de tres diferentes condiciones de almacenamiento de frutos sobre la germinación. Estos datos son esenciales para mejorar la producción de plantines de esta especie destinados a tareas de restauración ecológica y productiva, en áreas del Chaco semiárido afectadas por salinidad.

\section{MÉTODOS}

Recolección de frutos. Se recolectaron frutos maduros de bosques secundarios de P. ruscifolia, en la localidad de Maco, Departamento Capital, Provincia de Santiago del

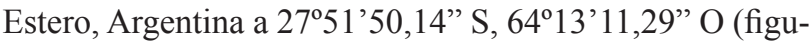
ra 1). Esta área se encuentra incluida en la región Chaqueña Occidental de Argentina. La cosecha se realizó durante los meses de diciembre de 2011 y enero de 2012. Se seleccionaron 10 ejemplares maduros y vigorosos. Del material 
cosechado se separaron los frutos destinados a la obtención de semillas para los ensayos de germinación y para el ensayo de almacenamiento a diferentes temperaturas.

Evaluación de tratamientos pregerminativos e interacción con el sustrato. Las semillas se extrajeron manualmente de los frutos maduros usando pinzas de corte, y se mantuvieron a $7 \pm 2{ }^{\circ} \mathrm{C}$ hasta su análisis para prevenir el ataque de insectos. Se realizó una selección previa descartando semillas que pudieran estar dañadas o vanas. Los ensayos se llevaron a cabo dos meses luego de la cosecha, en el Laboratorio de Semillas del Instituto de Silvicultura y Manejo de Bosques (INSIMA) perteneciente a la Facultad de Ciencias Forestales de la Universidad Nacional de Santiago del Estero. Se siguieron protocolos establecidos por ISTA (2015), para los ensayos de germinación se aplicaron los siguientes tratamientos pregerminativos: corte a nivel de cubierta seminal en el ápice distal de las semillas (opuesto al hilo; utilizando pinza de corte), abrasión colectiva de las semillas de cada repetición con discos de lija de granulometría $\mathrm{N}^{\circ} 80$ adheridos a la superficie de una caja de Petri con igual tiempo de procesamiento, inmersión en agua a temperatura ambiente $\left(25^{\circ} \mathrm{C}\right.$, durante 24 horas), inmersión en agua caliente a temperatura inicial de $100{ }^{\circ} \mathrm{C}$ (dejando enfriar progresivamente durante 24 horas previas a la siembra), inmersión en ácido sulfúrico $\left(\mathrm{H}_{2} \mathrm{SO}_{4}\right)$ concentrado ( $98 \mathrm{~g} \mathrm{~mol}^{-1}$, durante 3 minutos y posterior lavado durante 15 minutos en agua corriente para su neutralización), más un lote de semillas sin tratar, al que se consideró como control.
Antes de la siembra las semillas fueron desinfectadas con una solución de hipoclorito de sodio $\left(60 \mathrm{~g} \mathrm{~L}^{-1}\right)$ al $1 \%$ durante 5 minutos, enjuagándose posteriormente con agua destilada, durante 30 minutos. Los sustratos papel y arena se esterilizaron en autoclave antes de la siembra. La misma se realizó entre toallas de papel, a las cuales se agregó un volumen de agua destilada 2,5 veces su peso. La arena estéril se humedeció uniformemente con $125 \mathrm{~mL}$ de agua destilada, disponiendo $2 \mathrm{~kg}$ en cada contenedor. Para las muestras sembradas en papel así como para la siembra en arena, se utilizaron contenedores de poliestireno con tapas herméticas, de un volumen de $3.250 \mathrm{~mL}$.

Para las pruebas de germinación, se realizaron cuatro repeticiones de 50 semillas cada una por tratamiento. Se llevaron a cámara de germinación a $25^{\circ} \mathrm{C}$, con fotoperíodo de 8 horas de luz y 16 horas de oscuridad. Las evaluaciones se realizaron cada tres días, registrando el número de semillas germinadas que produjeron plántulas normales y anormales, número de semillas muertas y número de semillas duras. Las semillas se consideraron germinadas cuando los cotiledones emergieron de la cubierta seminal (germinación epígea) con desarrollo de plántula normal. La duración del ensayo fue de 21 días, considerando los antecedentes de Bravo et al. (2011) en Prosopis kuntzei Harms de la misma área de estudio.

Los ensayos de germinación se llevaron a cabo bajo un diseño factorial completamente aleatorizado. El análisis estadístico de datos se realizó con el programa Infostat (2017). El efecto de los tratamientos pregerminativos

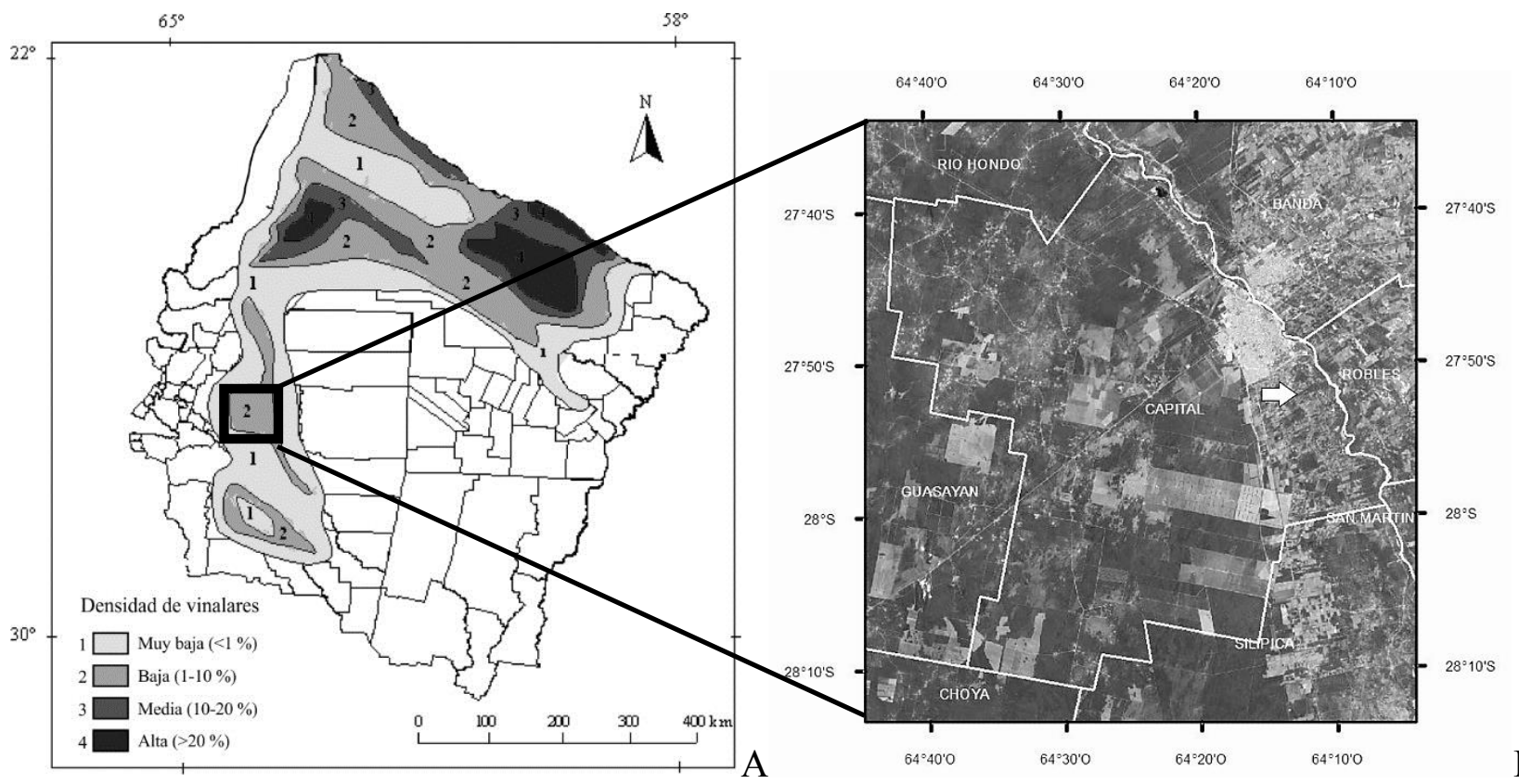

Figura 1.Distribución de Prosopis ruscifolia (vinal) en la Región Chaqueña de Argentina y área de recolección. A: distribución según grados de densidad tomado de Astrada y Adámoli (2005). B: Área de recolección de frutos en Santiago del Estero, Dpto. Capital.

Distribution area of Prosopis ruscifolia (vinal) in Chaco Region of Argentina and collection area. A: distribution according to degrees of density taken from Astrada and Adámoli (2005). B: Fruit collection area in Santiago del Estero, Capital Department. 
se evaluó por medio de Modelos Lineales Generalizados Mixtos (GLMM) con distribución binomial. Los tratamientos (control, corte, escarificado, agua fría, agua caliente y ácido) y los sustratos (papel y arena) se consideraron efectos fijos, y las repeticiones como aleatorios, tanto para el análisis de semillas germinadas con plántulas normales como para semillas no germinadas (duras y muertas). La significancia de los tratamientos, representados en este trabajo como la combinación de pretratamientos y sustratos, fueron determinadas a través de pruebas secuenciales y marginales (Wald), y los contrastes de los promedios fueron analizados con la prueba de Di Rienzo, Guzmán y Casanoves al 5 \%.

Efectos de diferentes temperaturas durante el almacenamiento de frutos. Para el estudio de las condiciones de almacenamiento se tomaron aleatoriamente ocho muestras de $100 \mathrm{~g}$ cada una y se secaron en estufa a $35^{\circ} \mathrm{C}$ hasta peso constante. Luego las muestras se almacenaron en envases herméticos de vidrio, en tres condiciones diferentes: a) control: temperatura ambiente $25 \pm 2{ }^{\circ} \mathrm{C}$, b) $7 \pm 2{ }^{\circ} \mathrm{C}$ y c) $-17 \pm 2{ }^{\circ} \mathrm{C}$.

El material de estudio se almacenó durante ocho meses hasta el inicio de los ensayos de germinación siguiendo protocolos generales de Reglas ISTA (2015), con cuatro repeticiones de 25 semillas cada una, por tratamiento. Como método pregerminativo se utilizó la escarificación química con ácido sulfúrico $\left(98 \mathrm{~g} \mathrm{~mol}^{-1}\right)$ siguiendo idéntico procedimiento al empleado en el ensayo de germinación y entre toallas de papel como sustrato. El ensayo de germinación posterior al almacenamiento de frutos a diferentes tempe- raturas tuvo una duración de 12 días y al final del mismo se registraron solo plántulas normales y semillas muertas. Bajo un diseño completamente aleatorizado, se evaluó el efecto de los diferentes tratamientos con modelos lineales generalizados mixtos (GLMM) con distribución binomial. Los tratamientos a bajas temperaturas y el control fueron considerados efectos fijos, y su significancia se determinó con las pruebas secuenciales y marginales (Wald).

Se determinó el porcentaje de germinación y para evaluar el vigor de las semillas en los distintos tipos de almacenamientos se consideraron dos índices: 1-índice de velocidad de germinación (IVG) (Maguire 1962), que expresa la velocidad en número de semillas germinadas por día, evaluándose con modelos lineales generales y mixtos; 2- el tiempo medio de germinación (TMG) (Nakagawa 1999), que mide la velocidad y dispersión de las semillas germinadas, estimado bajo modelos generalizados mixtos, con distribución binomial y enlace logit.

\section{RESULTADOS}

Evaluación de tratamientos pregerminativos e interacción con el sustrato. La escarificación con ácido sulfúrico fue el método más efectivo para estimular la germinación en semillas $P$. ruscifolia, ya que se obtuvo el mayor porcentaje de semillas germinadas con plántulas normales $(99 \%)$ sin diferencias significativas entre sustratos, pero con diferencias significativas de ambos respecto a los controles (cuadro 1). Los ensayos de germinación en ambos sustratos permitieron identificar plántulas normales y anormales y semillas duras y muertas de vinal a 21 días de

Cuadro 1. Porcentajes promedio de semillas germinadas con plántulas normales, plántulas anormales, semillas muertas y semillas duras, con diferentes tratamientos pregerminativos y diferentes sustratos.

Average percentages of seeds germinated with normal seedlings, abnormal seedlings, dead seeds and hard seeds, with different pregerminative treatments and different substrates.

\begin{tabular}{lccccc}
\hline Tratamiento pregerminativo & Sustrato & Plántulas normales & Plántulas anormales & Semillas muertas & Semillas duras \\
\hline Control & Arena & $16 \mathrm{e}$ & 0 & 0 & $84_{\mathrm{A}}$ \\
& Papel & $43 \mathrm{~d}$ & 0 & 1 & $56_{\mathrm{B}}$ \\
\multirow{3}{*}{ Ácido } & Arena & $98 \mathrm{a}$ & 0 & 2 & $0_{\mathrm{B}}$ \\
& Papel & $99 \mathrm{a}$ & 0 & 1 & $0_{\mathrm{B}}$ \\
Corte & Arena & $92 \mathrm{~b}$ & 0 & 8 & $0_{\mathrm{B}}$ \\
& Papel & $86 \mathrm{~b}$ & 4 & 10 & $0_{\mathrm{B}}$ \\
\multirow{3}{*}{ Escarificado } & Arena & $33 \mathrm{~d}$ & 0 & 4 & $63_{\mathrm{B}}$ \\
\multirow{5}{*}{ Agua caliente } & Papel & $37 \mathrm{~d}$ & 0 & 0 & $63_{\mathrm{B}}$ \\
& Arena & $69 \mathrm{~d}$ & 0 & 5 & $25_{\mathrm{B}}$ \\
Agua a temperatura ambiente & Papel & $50 \mathrm{c}$ & 2 & 3 & $45_{\mathrm{B}}$ \\
& Arena & $20 \mathrm{e}$ & 0 & 1 & $79_{\mathrm{A}}$ \\
& Papel & $9 \mathrm{e}$ & 0 & 0 & $91_{\mathrm{A}}$ \\
\hline
\end{tabular}

Medias con una letra común no son significativamente diferentes $(P>0,05)$. 
la siembra. La aparición de semillas germinadas en papel fue al cabo de tres días mientras que la aparición de los cotiledones en la siembra en arena se produjo a los seis días de la siembra. Se obtuvieron diferencias significativas en el porcentaje de semillas germinadas con plántulas normales y la interacción método* sustrato fue significativa $(P<0,0001$, cuadro 1$)$.

El número de semillas germinadas con plántulas normales con el método de corte de la cubierta seminal en el extremo distal de la semilla, no mostró diferencias significativas entre sustratos, pero si de ambos respecto a los controles. Sin embargo, presentó el mayor porcentaje de semillas muertas.

La inmersión en agua caliente utilizando arena como sustrato, mostró mayor porcentaje de germinación que en sustrato papel y hubo diferencias significativas entre ellos y respecto al control en arena.

La germinación de las semillas luego de la escarificación mecánica fue similar en los dos sustratos, sin diferencias significativas entre ellos, tampoco respecto al control en sustrato papel. Sin embargo, se observaron diferencias significativas respecto al control en arena.

La inmersión de las semillas en agua a temperatura ambiente produjo la menor proporción de plántulas normales, en los dos sustratos, sin diferencias estadísticamente significativas entre ambos, pero sí respecto al control en papel.

Todos los tratamientos para estimular la germinación produjeron un bajo porcentaje plántulas anormales y semillas muertas. El corte de la cubierta seminal en el extremo distal del embrión mostró el mayor porcentaje de plántulas anormales y de semillas muertas en papel. Sin embargo, no existe efecto significativo del método, del sustrato y de la interacción de ambos, bajo el análisis de las pruebas secuenciales y marginales $(P>0,99)$.

Las mayores proporciones de semillas duras se observaron con el método de inmersión en agua a temperatura ambiente (cuadro 1). La inmersión en agua caliente disminuyó significativamente el número de semillas duras, sin que existan tampoco diferencias entre sustratos. El método de escarificación mecánica es el segundo en orden de importancia en relación al número de semillas duras al final del ensayo, con igual tendencia respecto a los sustratos y al control en papel, que la mencionada para inmersión en agua fría. No se registraron semillas duras con los métodos de corte y ácido.

Efectos de diferentes temperaturas durante el almacenamiento de frutos. El inicio de la germinación se observó al tercer día desde la siembra (DDS), en las semillas cuyos frutos se almacenaron a $-17^{\circ} \mathrm{C}$ y al quinto DDS, para aquellas semillas cuyos frutos se almacenaron a $25{ }^{\circ} \mathrm{C}$ y a $7^{\circ} \mathrm{C}$ (figura 2). En el primer caso el $\mathrm{PG}$ al tercer día fue del $3 \%$, pero por razones de escala y tamaño del gráfico este valor no se representa en dicha figura.

Los porcentajes de germinación, según de los diferentes tratamientos se muestran en el cuadro 2 y representan la germinación de $P$. ruscifolia a 12 DDS. No hubo diferencias significativas en los porcentajes de germinación de semillas entre control y $-17^{\circ} \mathrm{C}$, por lo cual se compararon como un bloque con respecto a los resultados obtenidos del material almacenado a $7{ }^{\circ} \mathrm{C}$ se confirmó dichas diferencias $(P=0,0016)$.

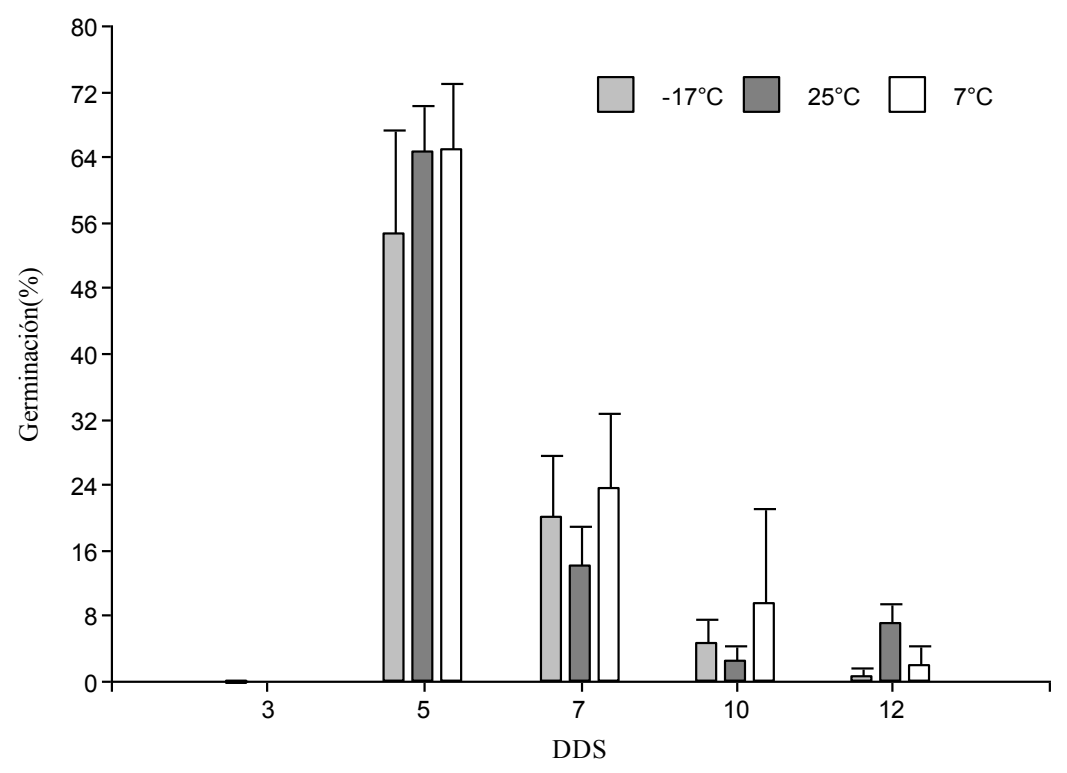

Figura 2. Porcentaje de germinación (no acumulado) de Prosopis ruscifolia en diferentes días desde la siembra (DDS), con distintas temperaturas de almacenamiento de frutos.

Germination percentage (not accumulated) of Prosopis ruscifolia on different days after sowing, at distinct fruit storage temperatures. 
Al finalizar el ensayo (12 DDS) no se observaron semillas duras en ninguno de los tratamientos, mientras que se detectó un $11 \%$ y $12 \%$ de semillas muertas en el control y $-17{ }^{\circ} \mathrm{C}$, respectivamente. Las bajas temperaturas de almacenamiento mejoraron el IVG y el TMG, sin diferencias significativas $(P<0,05)$ entre el almacenamiento a una temperatura de $-17^{\circ} \mathrm{C}$ y a una temperatura de $7^{\circ} \mathrm{C}$, pero significativamente diferentes al control (cuadro 2 ).

\section{DISCUSIÓN}

Germinación. Los resultados obtenidos en laboratorio para superar la dormición de las semillas de P. ruscifolia coinciden con los comunicados para otras especies de fabáceas, arbóreas y arbustivas, en las que se utilizaron diferentes métodos para estimular la germinación a través de desgaste, abrasión o interrupción de la continuidad y/o dureza de la cubierta seminal (Abraham de Noir et al. 2004, Baskin y Baskin 2014). Entre los tratamientos pregerminativos analizados, la inmersión en ácido sulfúrico muestra los mejores resultados, probablemente debido a la desintegración de la laminilla media de las macroesclereidas presentes en la cubierta seminal. Esta barrera de macroesclereidas es considerada responsable de las dificultades en la imbibición, en ausencia de algún método de escarificación (Villarreal Garza et al. 2013, Baskin y Baskin 2014). Pereira et al. (2016) observaron en Schinus areira L. que el ácido sulfúrico escarifica las paredes de las células tornándolas irregulares, acelerando la tasa de imbibición de las semillas. Las diferencias significativas en los porcentajes de germinación con este tratamiento pregerminativo, en relación a los controles en ambos sustratos, reafirman la presencia de una dormición física impuesta por la cubierta seminal en vinal.

Los resultados concuerdan con las diversas investigaciones en Prosopis realizadas por Villarreal Garza et al. (2013), Sobrevilla-Solís et al. (2013) y Rodríguez Araujo et al. (2017) quienes aplicaron el método de escarificación química con ácido sulfúrico para elevar el porcentaje de germinación en estas especies. El elevado porcentaje de germinación de semillas con plántulas normales y la baja incidencia de semillas muertas y duras obtenidos en este trabajo, sugieren que el tiempo de inmersión de 3 minutos en ácido sulfúrico es el apropiado para vinal. En este sentido, Sanabria et al. (2004) informaron un aumento en el porcentaje de germinación de Cassia moschata Kunth con la inmersión en ácido sulfúrico, disminuyendo además de 88 al $2 \%$ las semillas duras.

Los resultados indican que los controles producen un número significativamente menor de semillas germinadas, con diferencias entre sustratos. El mejor PG en el control se obtiene en papel, en concordancia con observado por Lopes de Oliveira et al. (2017) en Acacia auriculiformis A. Cunn. ex Benth., y a diferencia de lo señalado por Bravo et al. (2011) en Prosopis kuntzei, en la cual los porcentajes de semillas germinadas fueron mayores en arena.

En relación con los sustratos, el papel es uno de los más adecuados para realizar ensayos de germinación en laboratorio ya que está presente en la rutina de análisis de varias especies, permitiendo visualizar fácilmente el desarrollo de la raíz para una mejor la evaluación de las plántulas.

El sustrato arena puede presentar problemas en su uso relacionados a la distribución del tamaño de partícula y la capacidad de retención de agua, lo que puede causar un drenaje excesivo y sequedad de la superficie (Bravo et al. 2011). Otras dificultades que presenta la utilización de la arena como sustrato son la estandarización del peso volumétrico, el pH y la conductividad eléctrica (Trombin Souza et al. 2018).

La ausencia de diferencias significativas en la germinación, de las semillas tratadas con ácido sulfúrico entre arena y papel sugiere que ambos sustratos son apropiados para ensayos en laboratorio.

El corte en el extremo de la cubierta seminal como método para estimular la germinación en vinal, parece también apropiado con valores superiores al $85 \%$, aunque se observa una mayor incidencia de semillas muertas en ambos sustratos. Este último aspecto desfavorable podría atribuirse a pudriciones durante el curso del ensayo, producto de la interrupción abrupta de la continuidad de la cubierta seminal. Bravo et al. (2011) observaron en P. kuntzei un menor porcentaje de germinación con el método de corte en papel, atribuyéndolo a una excesiva acumulación de

Cuadro 2. Porcentaje promedio de germinación de semillas de Prosopis ruscifolia bajo diferentes temperaturas de almacenamiento de los frutos.

Average germination percentages of Prosopis ruscifolia seeds after different fruit storage temperatures.

\begin{tabular}{ccccc}
\hline $\begin{array}{c}\text { Temperatura de } \\
\text { almacenamiento }\end{array}$ & $\begin{array}{c}\text { Inicio de la germinación } \\
\text { (DDS) }\end{array}$ & $\begin{array}{c}\text { Germinación } \\
(\%)\end{array}$ & $\begin{array}{c}\text { Índice de velocidad de } \\
\text { germinación }\end{array}$ & $\begin{array}{c}\text { Tiempo medio de } \\
\text { germinación }\end{array}$ \\
\hline $25^{\circ} \mathrm{C}$ & 5 & $89 \pm 2 \mathrm{~b}$ & $7,93 \mathrm{~b}$ & $6,23 \mathrm{~b}$ \\
$7{ }^{\circ} \mathrm{C}$ & 5 & $99 \pm 1 \mathrm{a}$ & $8,75 \mathrm{a}$ & $6,08 \mathrm{a}$ \\
$-17^{\circ} \mathrm{C}$ & 3 & $88 \pm 4,9 \mathrm{~b}$ & $8,21 \mathrm{a}$ & $5,53 \mathrm{a}$ \\
\hline
\end{tabular}

Medias con una letra común no son significativamente diferentes $(P>0,05)$. 
agua alrededor de las semillas y a una baja aireación entre las toallas de papel. La ausencia de semillas duras con el método de corte, en ambos sustratos, reafirman la dormición impuesta por la cubierta seminal en $P$. ruscifolia.

Los porcentajes de germinación obtenidos con escarificación mecánica con lija en ambos sustratos son significativamente menores a los obtenidos con los métodos de inmersión en ácido sulfúrico, corte de la cubierta seminal e inclusive sin diferencias significativas respecto al control en papel. El elevado porcentaje de semillas duras al finalizar el ensayo indica que este método es insuficiente para superar la barrera física de la cubierta seminal, ya que las semillas escarificadas sembradas en ambos sustratos no mostraron diferencias significativas respecto al control en papel. Sin embargo, Sobrevilla-Solís et al. (2013) propusieron la escarificación mecánica como un método adecuado para estimular la germinación en Prosopis, extremando cuidados para no afectar la calidad fisiológica de las semillas (Dutra et al. 2007) Además, Rodríguez Araujo et al. (2017) indicaron que la técnica de escarificación puede variar según la persona que la realiza, obteniendo con estos métodos elevados porcentajes de germinación en distintas especies de Prosopis. Por lo tanto, esto podría explicar los altos porcentajes de semillas duras obtenidas en la presente investigación. Sin embargo, según de Menezes Silva et al. (2011) la escarificación con lija resultó el método más eficiente en Sesbania virgata (Cav) Pers. comparado con la inmersión en ácido sulfúrico. La distribución de la humedad propia del riego inicial es probablemente diferente entre las partículas de arena que en papel.

Los porcentajes de semillas germinadas con plántulas normales fueron mayores con el método de inmersión en agua caliente que en agua a temperatura ambiente y existen diferencias significativas entre sustratos solo en el primero. Esto podría sugerir la existencia de inhibidores químicos termolábiles a nivel de cubierta seminal o algún tipo de daño al embrión. Es común la presencia de inhibidores de naturaleza hidrofóbica como cutinas, suberinas, pectinas, lípidos y ligninas (Ferreira y Borghetti, 2004). Los resultados obtenidos con inmersión en agua caliente sugieren la eliminación de inhibidores de naturaleza lipídica, que no produce la inmersión en agua a temperatura ambiente. Lopes de Oliveira et al. (2017) determinaron que la inmersión en agua a temperatura ambiente no permite superar la barrera impuesta por la cubierta seminal a la imbibición.

Almacenamiento. Los elevados porcentajes de germinación observados en el ensayo de almacenamiento indican el excelente vigor del lote de semillas. Los resultados sugieren que almacenamiento de frutos de $P$. ruscifolia a 7 ${ }^{\circ} \mathrm{C}$ permiten un óptimo porcentaje de germinación en esta especie. Bonner (2008) describió el comportamiento de las semillas de especies de Prosopis como ortodoxas, en congruencia con resultados del presente trabajo. Valores de porcentaje de germinación semejantes a los observados en este trabajo, se comunicaron para las semillas ortodoxas de
Anadenanthera colubrina (Vell.) Bren almacenadas bajo condiciones similares (Rojas Espinoza 2004). Los resultados obtenidos con el almacenamiento a $-17{ }^{\circ} \mathrm{C}$ podrían representar una alternativa más segura, sobre todo cuando se almacenan frutos completos ya que podría reducir el ataque de insectos (Mazzuferi y Conles 2004).

El incremento en IVG y TMG en semillas provenientes de frutos almacenados en condiciones de bajas temperaturas, podría atribuirse a una mejor conservación del sistema enzimático que participa en la movilización de azúcares (Azadi y Younesi, 2013). Si bien el contenido de humedad no ha sido considerado en este trabajo, el almacenamiento a temperatura ambiente de los frutos de vinal podría haber generado un envejecimiento en el lote de semillas, justificando el menor IVG y el mayor TMG obtenido durante el ensayo.

Debido a que las legumbres de especies del género Prosopis contienen un elevado porcentaje de azúcares (González Galán et al. 2008), los estudios en relación a las condiciones óptimas para el almacenamiento son útiles y necesarios para viveristas.

\section{CONCLUSIONES}

Los resultados de este trabajo identifican que la inmersión en ácido sulfúrico es el mejor método para estimular la germinación de semillas en $P$. ruscifolia y el papel, el sustrato más adecuado para la obtención de plántulas normales en ensayos de laboratorio. Este resultado permitirá optimizar las evaluaciones de calidad de semillas para esta especie. Se confirma la necesidad de escarificación para romper la dormición.

El almacenamiento de frutos de vinal a $7{ }^{\circ} \mathrm{C}$ es el método más apropiado para alcanzar los mayores porcentajes de germinación. Esta información es de importancia para viveristas, ya que confirma la posibilidad de almacenamiento de frutos de P. ruscifolia, en medios de baja tecnología y disminuye la urgencia de las tareas de limpieza y liberación de semillas. El alto porcentaje de germinación de semillas provenientes de frutos almacenados a $-17^{\circ} \mathrm{C}$ indica tolerancia de las mismas a temperaturas bajo cero. Se plantea la necesidad de continuar las pruebas considerando periodos de almacenamiento más prolongados.

\section{REFERENCIAS}

Abraham de Noir F, S Bravo. 2014. Frutos de leñosas nativas de Argentina, parte I Chaco occidental y serrano. Santiago del Estero, Argentina. Universidad Nacional de Santiago del Estero - UNSE, Facultad de Ciencias Forestales. 194 p. EBook. ISBN 978-987-1676-19-4.

Abraham de Noir F, M Gulotta, R Abdala. 2004. How to improve germination in Caesalpinia paraguariensis Burk. Seed Science and Technology 32(1): 235-238. DOI: https://doi. org/10.15258/sst.2004.32.1.26

Astrada E, J Adámoli. 2005. Ecología y manejo de vinalares: Perspectiva regional y aplicaciones en el centro de Formo- 
sa. In Arturi MF, JL Frangi, JF Goya eds. Ecología y manejo de bosques de Argentina. La Plata, Argentina. Editorial de la Universidad Nacional de La Plata. 23 p.

Azadi MS, E Younesi. 2013. The effects of storage on germination characteristics and enzyme activity of sorghum seeds. Journal of Stress Physiology \& Biochemistry 9(4): 289-298.

Baskin JM, JM Baskin. 2014. Seeds Ecology, biogeography, and evolution of dormancy and germination. $2^{\mathrm{a}}$ ed. San Diego, California, USA. Academic Press. 1573 p.

Bonner FT. 2008. Storage of seeds. In Bonner FT, RP Karrfalt eds. The Woody Plant Seed Manual. Chapter 4. Washington DC, USA. US Department of Agriculture. Handbook 727 p. 85-95.

Bravo S, R Abdala, F Abraham, M Pece. 2011. Treatments to Improve Germination of Prosopis kuntzei. Seed Technology Journal 33(1): 55-62.

de Menezes Silva PE, EF Santiago, D de Menezes Daloso, E Marques da Silva, J Oliveira Silva. 2011. Treatments to break dormancy in Sesbania virgata (Cav.) Pers seeds. Idesia 29(2): 39-45.

Dousseau S, AA de Alvarenga, R Mendes Guimarães, T Silva, L Telde, N Custódio, I de Souza Chaves. 2011. Ecofisiologia da germinação de sementes de Campomanesia pubescens. Ciência Rural 41(8): 1362-1368. DOI: 10.1590/S010384782011000800011

Dutra AS, S Medeiros Filho, EM Teófilo, FO Diniz. 2007. Seeds Germination of Senna siamea (Lam.) H.S. Irwin e Barneby-Caesalpinoideae. Brazilian Journal of Seeds 29: 160-164. DOI: http://dx.doi.org/10.1590/S010131222007000100022

Felker P, M Ewens, H Ochoa. 2000. Environmental influences on grafting success of Prosopis ruscifolia (vinal) onto Prosopis alba (algarrobo blanco). Journal of Arid Environments 46(4): 433-439.

Ferreira AG, F Borghetti. 2004. Germinação: do básico ao aplicado. Porto Alegre, Brasil. Artmed. 323 p.

González Galán A, A Duarte Corrêa, CM Patto de Abreu, MF Piccolo Barcelos. 2008. Caracterización química de la harina del fruto de Prosopis spp. procedente de Bolivia y Brasil. Archivos Latinoamericanos de Nutrición 58(3): 309-315 .

InfoStat versión 2017. Grupo InfoStat, FCA, Universidad Nacional de Córdoba. Córdoba, Argentina. https://www.researchgate.net/publication/319875201 Grupo InfoStat FCA Universidad Nacional de Cordoba Argentina

ISTA (International Seed Testing Association, CH). 2015. International Rules for Seed Testing. Zurich, Swittzerland. ISAT. 8 p.

Lopes De Oliveira ER, J Correia De Araujo Neto, C Barboza Da Silva, V Marques Ferreira, LF Gomes Chaves, MI Rodrigues Silva Das Neves. 2017. Overcoming dormancy and germination requirements for Acacia auriculiformis A. Cunn Ex. Benth seeds. Asian Academic Research Journal of Multidisciplinary 4 (12):17.

Maguire JD. 1962. Speed of germination-aid in selection and evaluation for seedling emergence and vigor. Crop Science 2(2): 176-177. DOI: https://doi.org/10.2135/cropsci1962.0 011183X000200020033x

Mazzuferi V, M Conles. 2005. Insectos y hongos que afectan las semillas de Prosopis. In Verzino GE, MJ Joseau eds. El Banco Nacional de Germoplasma de Prosopis. Córdoba, Argentina. Facultad de Ciencias Agropecuarias, Universi- dad Nacional de Córdoba. p. 69-78.

Meloni DA, MR Gulotta, CA Martínez. 2008. Prosopis ruscifolia Griseb. (vinal) tolera concentraciones salinas equivalentes al agua de mar y excluye iones tóxicos de la parte aérea. Quebracho 16: 32-40.

Muñoz BC, JA Sánchez, LA Montejo, y González, J Reino. 2009. Valoración germinativa de 20 accesiones de leguminosas almacenadas en condiciones desfavorables. Pastos y forrajes 32(3): 1-15.

Nakagawa J. 1999. Testes de vigor baseados no desempenho das plântulas. In Krzyzanowski FC, RD Vieira, JB França-Neto eds. Vigor de sementes: conceitos e testes. Londrina, Brasil. Abrates. Cap. 2. p. 2.1-2.24.

Pereira MP, F Corrêa, M Polo, EM de Castro, AÁ Cardoso, FJ Pereira. 2016. Seed germination of Schinus molle L. (Anacardiaceae) as related to its anatomy and dormancy alleviation. Seed Science Research 26(4): 351-361. DOI: $10.1017 /$ S0960258516000167.

Piotto B, E Fallieri. 2001. La conservazione dei semi. In Piotto B, A Di Noi eds. Propagazione per seme di alberi e arbusti della flora Mediterranea. Rome, Italy. Agenzia Nazionale per la protezione dell'Ambiente (ANPA). p. 89-97. Disponible en: https://www.isprambiente.gov.it/ contentfiles/00003500/3510-propagazione-per-seme.pdf

Rodríguez Araujo ME, DR Pérez, GL Bonvissuto. 2017. Seed germination of five Prosopis shrub species (FabaceaeMimosoideae) from the Monte and Patagonia phytogeographic provinces of Argentina. Journal of Arid Environments 147: 159-162. DOI: https://doi.org/10.1016/j.jaridenv.2017.07.019.

Rojas Espinoza E. 2004. Desiccation and storage of Anadenanthera colubrina seeds. In Sacando M, D Jøker, ME Dulloo, KA Thompsen eds. Comparative storage biology of tropical tree seeds. Rome, Italy, Internacional Plant Genetic Resources Institute. p. 251-257.

Sanabria D, R Silva-Acuña, M Oliveros, U Manrique. 2004. Germinación de semillas de las leguminosas arbustivas forrajeras Cratylia argentea y Cassia moschata sometidas a inmersión en ácido sulfúrico. Bioagro 16 (3): 225-230.

Sobrevilla-Solís JA, M López-Herrera, AL López-Escamilla, L Romero-Bautista. 2013. Evaluación de diferentes tratamientos pregerminativos y osmóticos en la germinación de semillas Prosopis laevigata (Humb. et Bonpl. Ex Willd) M. C. Johnston. In Pulido-Flores G, S Monks eds. Estudios científicos en el estado de Hidalgo y zonas aledañas, Volumen II. p. 83-95.

Taleisnik E, D López Launestein. 2011. Leñosas perennes para ambientes afectados por salinidad. Una sinopsis de la contribución argentina a este tema. Ecología Austral 21:3-14.

Trombin Souza M, M Trombin, M Panobianco. 2018. Morphological characterization of fruit, seed and seedling, and seed germination test of Campomanesia guazumifolia. Journal of Seed Science 40(1): 75-81. https://dx.doi. org/10.1590/2317-1545v40n1186143

Villagra PE, A Vilela, C Giordano, J Álvarez. 2010. Ecophysiology of Prosopis species from the arid lands of Argentina: what do we know about adaptation to stressful environments? In Ramawat KG ed. Desert Plants. Biology and Biotechnology. Heidelberg, Germany. Springer-Verlag. p 321-340.

Villagra P, J Álvarez. 2019. Determinantes ambientales y desafíos 
para el ordenamiento forestal sustentable en los algarrobales del Monte, Argentina. Ecología Austral 29:146-155.

Villareal Garza JA, A Rocha Estrada, ML Cardenas-Avila, S Moreno Limon, M Gonzalez Alvarez, V Vargas López. 2013. Caracterizacion morfometrica, viabilidad y germinación de semillas de mezquite y huizache en el Noeste de México. FYTON 82: 169-174.
Were J, N Munjuga, MI Daws, N Motete, D Erdey, D Baxter, P Berjak, HW Pritchard, C Harris, CA Howard. 2004. Desiccation and storage of Prunus africana seeds (Hook. f.) Kalkm. In Sacando M, D Jøker, ME Dulloo, KA Thompsen eds. Comparative storage biology of tropical tree seeds. Rome, Italy. Internacional Plant Genetic Resources Institute. p. 67-74.

Recibido; 17/12/19

Aceptado: 20/04/20 
